\title{
LETTER TO THE EDITORS
}

\section{Paraformaldehyde Fixation and Some Characteristics of Lens Proteins as Measured by Raman Microspectroscopy}

In previous studies (Huizinga et al., 1989; Bot et al., 1989 ) it was demonstrated that the results of water content measurements of human and rabbit lenses estimated by Raman microspectroscopy were comparable to those using differential scanning calorimetry, nuclear magnetic resonance spectroscopy, dynamic light scattering and the dry-weight-frozensection technique. It was emphasized that Raman microspectroscopy is superior in detecting local variations in water content. It was further demonstrated that fixation of the lenses with a cacodylate buffered paraformaldehyde solution had only minor quantitative effects on the water content. Finally, it was shown that slicing of the fixed lenses further improved local positioning of the Raman probe and enabled Raman analysis of adult and aged human lenses.

The role of the conversion of sulfhydryl (SH) groups into disulfide (SS) bonds in the formation of high molecular weight (HMW) proteins, and consequently in cataractogenesis, seems well established (East et al., 1978; Ozaki et al., 1983; Spector, 1984; Yu et al., 1985). In addition it was found, in aging mice lenses, that in concert with the decrease in SH-groups the ratio of the tryptophan doublet $\left(I_{855} / I_{760}\right)$ also decreases, whereas the tyrosine doublet $\left(I_{855} / I_{832}\right)$ remains constant (Ozaki et al., 1983). In a hereditary nuclear cataract in mice (cac strain) a decrease in both the tryptophan and tyrosine doublet was found (Itoh et al., 1983). These declines appeared concomitant with the first clinical signs of nuclear opacification. In view of this evidence it seems likely that the SH to SS conversion and the changes in the ratio of the tryptophan and tyrosine doublets play a role in the formation of HMW proteins and cataractogenesis. Because an important goal of our Raman studies is the analysis of changes in water content and protein composition of aging human lenses and as adult human lenses can only be studied by Raman spectroscopy after paraformaldehyde fixation and subsequent slicing, it was essential to analyze the effects of paraformaldehyde fixation on the SH-groups and the tryptophan and tyrosine doublets.

For this study fresh rabbit lenses (Chinchilla strain, 6 months old) were screened along the visual axis using the Raman microspectroscopic procedure described earlier (Gijsbers et al., 1986). Subsequently the lenses were immersed in a $0.08 \mathrm{M}$ cacodylate buffered $1 \%$ paraformaldehyde solution (310 mOsmol, $\mathrm{pH} 7 \cdot 3$ ). After 1 week the lenses were remeasured. The SH-content was indicated as the ratio $I_{2580} / I_{2731}$ (SH/protein) and the tryptophan doublet (ratio: $I_{880} / I_{760}$ ) and the tyrosine doublet (ratio: $I_{855} / I_{832}$ ) were indicated according to Ozaki et al. (1983). The differences between the lenses under investigation,

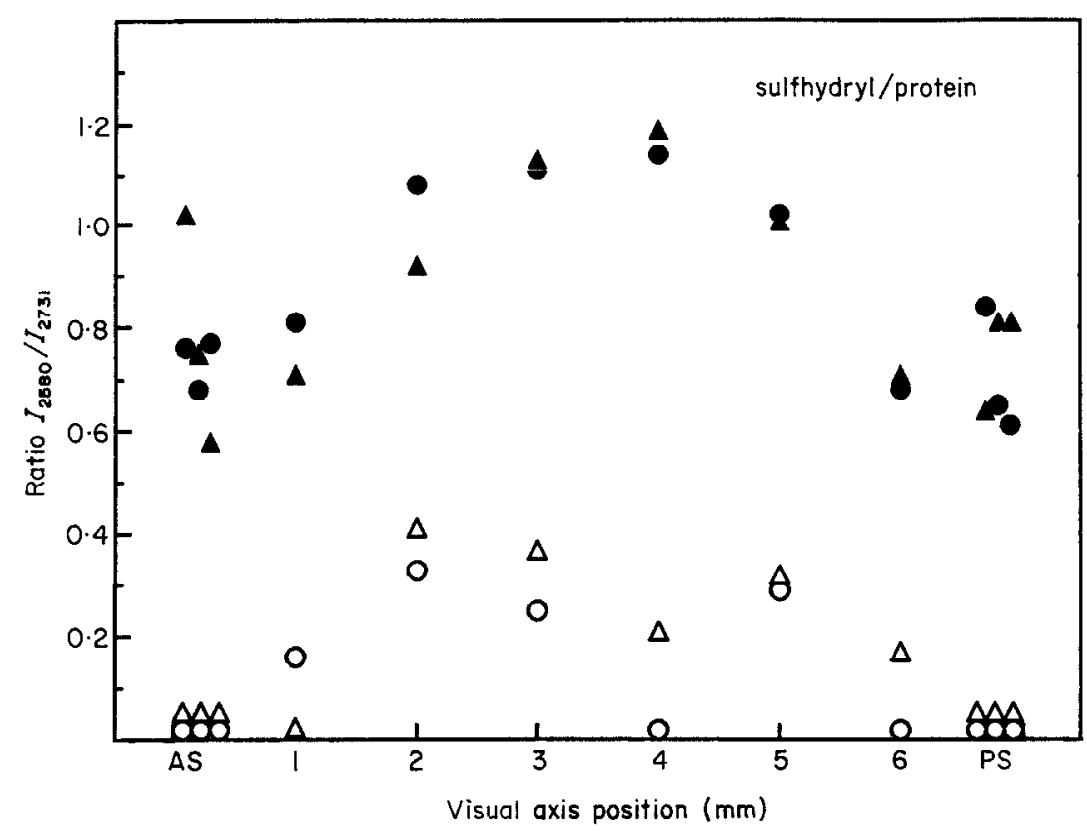

FIG. 1. Raman intensity ratios of sulfhydryl $\left(I_{2580} \mathrm{~cm}^{-1}\right) /$ protein $\left(I_{2731} \mathrm{~cm}^{-1}\right)$ along the visual axis of two (triangles, circles) rabbit lenses prior to (closed symbols) and after (open symbols) fixation with paraformaldehyde. $t$-Statistics revealed no significant difference between the two fresh lenses. The difference between the fixed and unfixed measurements was highly significant. AS: anterior surface. PS: posterior surface. 


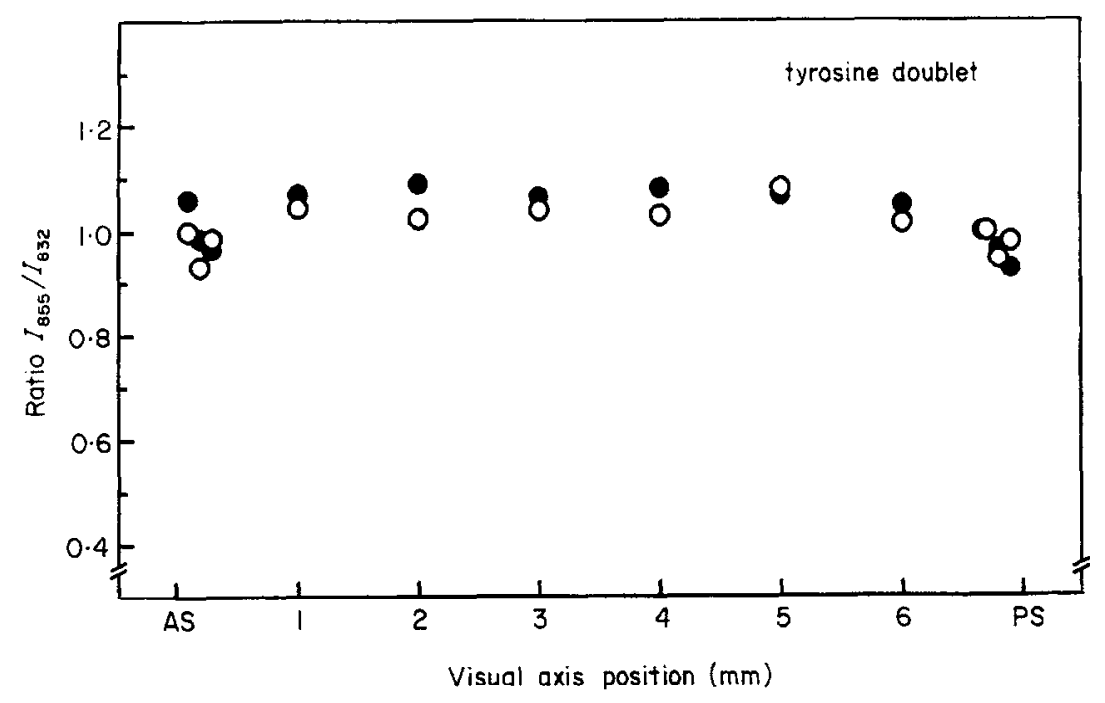

FIG. 2. Raman intensity ratio of the tyrosine doublet at $I_{855} \mathrm{~cm}^{-1}$ and $I_{832} \mathrm{~cm}^{-1}$ along the visual axis of rabbit lenses prior to $(O)$ and after $(O)$ paraformaldehyde fixation. As $t$-statistics revealed no significant differences between the two lenses before and after fixation ( $t$-values: 0.42 and $1 \cdot 83$, respectively) the combined means of the two lenses are indicated. Least square regression analysis revealed a significantly higher ratio in the nucleus. AS: anterior surface. PS: posterior surface.

regarding the fresh and fixed Raman characteristics, were tested using $t$-statistics, and the visual axis distributions were tested using least square regression analysis. The results of the present study are summarized in the Figs 1-3.

As indicated in Fig. 1, $t$-statistics revealed no significant difference between the two fresh lenses regarding the $\mathrm{SH} /$ protein ratio, whereas the difference between fresh and fixed lenses was highly significant. This means that paraformaldehyde fixation leads to a dramatic and highly significant decrease in the ratio $I_{2580} / I_{2731}$. As paraformaldehyde does not affect the protein content (Bot et al., 1989) this decrease must be the result of a decrease in the SH-stretch Raman signal. This decrease is due to the formation of semithioacetal groups and methylene bridges between SH and paraformaldehyde (cf. Pearse, 1968). A practical consequence of this result is that, without further precautions (e.g. the alkylation of SH-groups yielding a product with a distinctive Raman signal, in paraformaldehyde fixed lenses) the $\mathrm{SH}$ to $\mathrm{SS}$ conversion cannot be deduced on account of the $I_{2580}$ Raman intensity. However, as it is unlikely that the SS-bonds are reacting with the paraformaldehyde (cf. Pearse, 1968) the SS-Raman signal at $I_{508}$ will be a reliable indication of the SH to SS conversion. However, in young rabbit lenses the $I_{508}$ signal of SSbonds is extremely low and could not be verified this in the present study. Studies in old human lenses, having more pronounced SS-levels, are in progress to verify

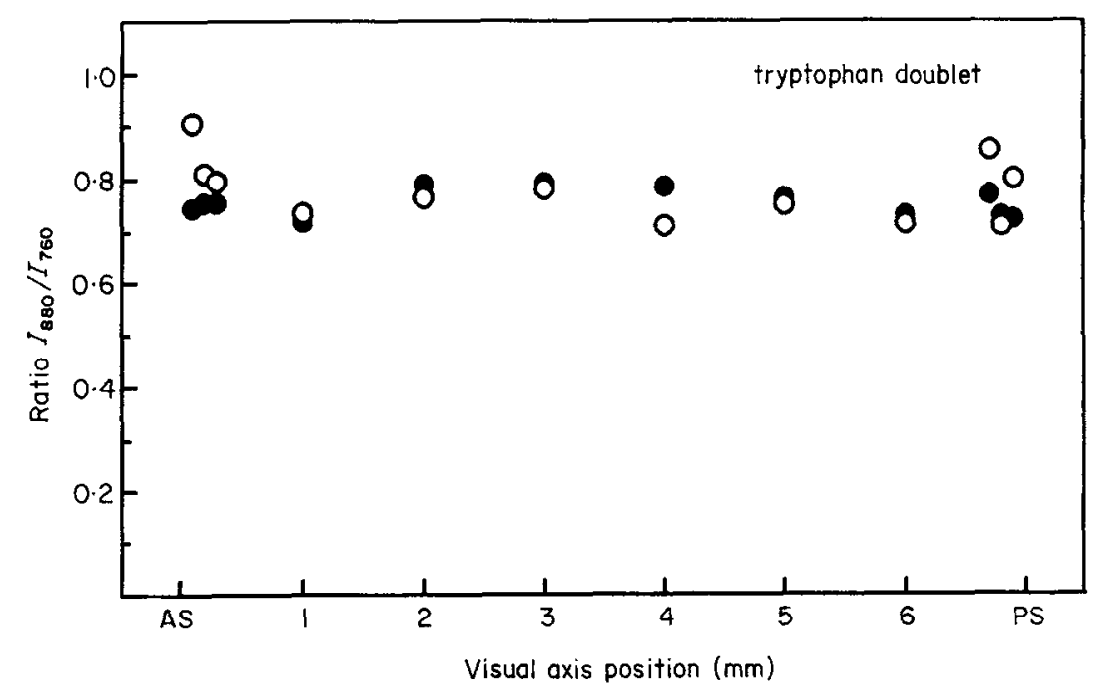

Fig. 3. Raman intensity ratio of the tryptophan doublet at $I_{880} \mathrm{~cm}^{-1}$ and $I_{780} \mathrm{~cm}^{-1}$ along the visual axis of rabbit lenses prior to $(O)$ and after $(O)$ fixation with paraformaldehyde. As $t$-statistics revealed no significant differences between the two lenses measured the combined means of the two lenses are indicated. The overall difference between fresh and fixed lenses is not significant $(t=1.66 ; P \gg 0.05)$. If the three most anterior and three most posterior positions are considered there proved to exist a slight but significant increase after paraformaldehyde fixation $(t=3.64 ; P<0.01)$ AS: anterior surfacc, PS: posterior surface. 
this supposition. Figures 2 and 3 clearly indicate that paraformaldehyde had no significant overall effect on the ratios of the tyrosine and tryptophan doublets, and that paraformaldehyde fixed lenses can be used to analyze changes in these aromatic amino acids during aging and cataract formation. With respect to the tryptophan doublet, the ratio has slightly increased in the most anterior and posterior positions after fixation.

The data shown in Fig. 1 could be satisfactorily represented with a parabolic distribution. This distribution of SH-groups along the visual axis in fresh lenses (Fig. 1), with a significantly higher Raman signal in the lens nucleus, corresponds to the results by Askren, Yu and Kuck (1979) and Yu et al. (1985). in young rat lenses, and in guinea-pig and human lenses. It indicates that the nucleus of these mammalian lenses has a relatively high content of sulfhydryl groups. As outlined by Siamwiza et al. (1975), the intensity ratio of the tyrosine doublet $\left(I_{855} / I_{832}\right)$ is related primarily to the state of the phenolic hydroxyl group and changes in relation to the 'buried' or 'exposed' position of the tyrosine

a Netherlands Ophthalmic Research Institute,

P. O. Box 12141,

1100 AC Amsterdam,

The Netherlands

${ }^{b}$ Twente University of Technology,

Faculty of Applied Physics,

P.O. Box 217,

7500 AE Enschede,

The Netherlands within the protein molecule: being lower in the 'buried' or 'masked' position than in the 'exposed' position. According to Tu (1982), the relative amount of 'buried' and 'exposed' tyrosine residues can be calculated once the total number of tyrosine residues is known. The tyrosine data summarized in Fig. 2 show a small but significant increase in the $I_{855} / I_{832}$ ratio towards the nucleus, indicating that in the rabbit lens there is an increase in the number of exposed tyrosine molecules from cortex to nucleus. Kitagawa, Azuma and Hamaguchi (1979) have emphasized the relevance of the $I_{880}$ signal of tryptophan as an indicator of the 'buried' or 'exposed' position of the molecule within the protein: being higher when 'buried'. In the fresh lenses there is a slight tendency towards an increase in the nucleus indicating more 'buried' tryptophan residues in the nucleus than in the cortex. However, the slight cortical increase after fixation and the variations in the nuclear region have to be analyzed in more detail before this conclusion can be definite.

(Received 2 August 1989 and accepted in revised form 25 October 1989)

* For correspondence.

\section{References}

Askren, C. C., Yu, N.-T. and Kuck. J. F. R. Jr. (1979). Variation of the concentration of sulfhydryl along the visual axis of aging lenses by laser Raman optical dissection technique. Exp. Eye Res. 29, 647-54.

Bot, A. C. C., Huizinga, A., De Mul, F. F. M., Vrensen, G. F. J. M. and Greve, J. (1989). Raman microspectroscopy of fixed rabbit and human lenses and lens slices: new potentialities. Exp. Eye Res. 49, 161-9.

East, E. J., Chang, R. C. C., Yu, N.-T. and Kuck, J. F. R. Jr. (1978). Raman spectroscopic measurement of total sulfhydryl in intact lens as affected by aging and ultraviolet irradiation. J. Biol. Chem. 253, 1436-41.

Gijsbers. G., Vrensen. G.. Willekens, B., Maatman. D.. De Mul, F. and Greve, J. (1986). Raman microspectroscopic investigations of human eye lenses. In Laser Scattering Spectroscopy of Biological Objects (Eds Stepanek, J., Anzenbacher, P. and Sedlacek, B.). Pp. 583-94. Elsevier: Amsterdam.

Huizinga, A., Bot, A. C. C., De Mul, F. F. M., Vrensen, G. F. J. M. and Greve, J. (1989). Local variation in absolute water content of human and rabbit eye lenses measured by Raman microspectroscopy. Exp. Eye Res. $48,487-96$.

Iriyama, K. (1983). Raman spectroscopic study of age related structural changes in the lens proteins of an intact mouse lens. Biochemistry 22, 6254-9.
Itoh, K., Ozaki, Y., Mizuno, A. and Iriyama, K. (1983). Structural changes in the lens proteins of hereditary cataracts monitored by Raman spectroscopy. Biochemistry 22, 1773-8.

Kitagawa, T., Azuma, T. and Hamaguchi, K. (1979). The Raman spectra of Bence-Jones proteins: disultide stretching frequencies and dependence of Raman intensity of tryptophan residues on their environments. Biopolymers 18, 451-65.

Ozaki, Y., Mizuno, A., Itoh, K., Yoshiura, M., Iwamoto, T. and Iriyama, K. (1983). Raman spectroscopic study of age-related structural changes in the lens proteins of an intact mouse lens. Biochemistry 22, 6254-9.

Pearse, A. G. E. (1968). In Histochemistry: Theoretical and Applied. Vol. I. Pp. 73. Churchill Livingstone: Edinburgh.

Siamwiza, M. N., Lord, R. C., Chen, M. C., Takamatsu, T., Harada, I., Matsuura, H. and Shimanouchi, T. (1975). Interpretation of the doublet at 850 and $830 \mathrm{~cm}^{-1}$ in the Raman spectra of tyrosyl residues in proteins and certain model compounds. Biochemistry 14, 4870-6.

Spector, A. (1984). The search for a solution to senile cataracts. Invest. Ophthalmol. Vis. Sci. 25, 130-40.

Tu, A. T. (1982). Raman Spectroscopy in Biology: Principles and Applications; Chapter 3, Proteins. Wiley: New York.

Yu, N.-T., DeNagel, D. C., Pruett, P. L. and Kuck, J. F. R., Jr. (1985). Disulfide bond formation in the eye lens. Proc. Natl. Acad. Sci. U.S.A. 82, 7965-8. 